\title{
Stereoselective Vinylogous Mukaiyama Aldol Reaction of $\alpha$-Haloenals
}

\author{
Yoichi Iwasaki, Ryosuke Matsui, Takahiro Suzuki, Atsuo NaKazaki, and Susumu Kobayashi* \\ Faculty of Pharmaceutical Sciences, Tokyo University of Science; 2641 Yamazaki, Noda, Chiba 278-8510, Japan. \\ Received January 11, 2011; accepted January 27, 2011; published online January 31, 2011
}

We have developed a high-yielding and stereoselective vinylogous Mukaiyama aldol reaction (VMAR) of $\alpha$ haloenals. Contrary to the simple $\alpha, \beta$-unsaturated aldehyde, $\alpha$-haloenals were found to be reactive affording the corresponding VMAR adducts in excellent yields. Some transformations of VMAR adducts by Pd-mediated cross-coupling were also examined in order to demonstrate the synthetic utility of VMAR of $\alpha$-haloenals.

Key words vinylogous Mukaiyama aldol reaction; $\alpha$-haloenal; stereoselectivity; cross-coupling

We previously reported a highly stereoselective vinylogous Mukaiyama aldol reaction (VMAR) using vinylketene silyl $\mathrm{N}, \mathrm{O}$-acetal $\mathbf{1}$ and $\mathbf{2}$, which provides a unique and remarkable entry to a remote asymmetric induction (Chart 1$){ }^{1-3)}$ From a synthetic point of view, this method can directly afford the anti- $\delta$-hydroxy- $\alpha, \gamma$-dimethyl- $\alpha, \beta$-unsaturated carbonyl unit which is seen in many naturally occurring products. Indeed, VMAR has successfully been utilized in natural product syntheses by many groups ${ }^{4-20)}$ including ourselves. ${ }^{21-25)}$ However, $\alpha, \beta$-unsaturated aldehyde is not generally a good substrate for VMAR in terms of yield (i.e., low to moderate). High yield could be achieved by the addition of a catalytic amount of water, ${ }^{3)}$ or by carrying out the reaction for a prolonged period of time. ${ }^{5,11)}$ In this context, we became interested in the employment of $\alpha$-haloenals as substrates which are considered to be much more reactive compared to simple enals. We also reasoned that the VMAR products from $\alpha$ haloenals could serve as versatile intermediates for introducing a variety of substituents using well-established Pd-mediated methodologies. Herein, we report a high-yielding and stereoselective VMAR of $\alpha$-haloenals achieved under standard conditions ( $\mathrm{TiCl}_{4}$ in $\mathrm{CH}_{2} \mathrm{Cl}_{2}, 2.0$ eq of aldehyde). Some transformations of VMAR products from $\alpha$-haloenals by Pdcatalyzed cross-coupling are also described.

In this study, we examined VMAR of $\alpha$-iodoenal $\mathbf{3 a},{ }^{26)} \alpha$ bromoenal $\mathbf{3 b},{ }^{27)}$ and $\alpha$-chloroenal $\mathbf{3} \mathbf{c}^{28,29)}$ (Table 1). According to the established protocol, VMAR of $\alpha$-iodoenal $\mathbf{3 a}$ $(2.0 \mathrm{eq})$ with the vinylketene silyl $N, O$-acetal 2 using $\mathrm{TiCl}_{4}$ $(1.0 \mathrm{eq})$ afforded the corresponding anti-aldol adduct $\mathbf{4 a}$ in excellent yield with high diastereoselectivity (entry 1 ). In addition, VMAR of $\alpha$-bromoenal $\mathbf{3 b}$ and $\alpha$-chloroenal $\mathbf{3 c}$ also provided anti-adducts $\mathbf{4 b}$ and $4 \mathrm{c}^{30}$ in excellent yield, respectively (entries 2,3 ). Notably, $\alpha$-halo substituents improved

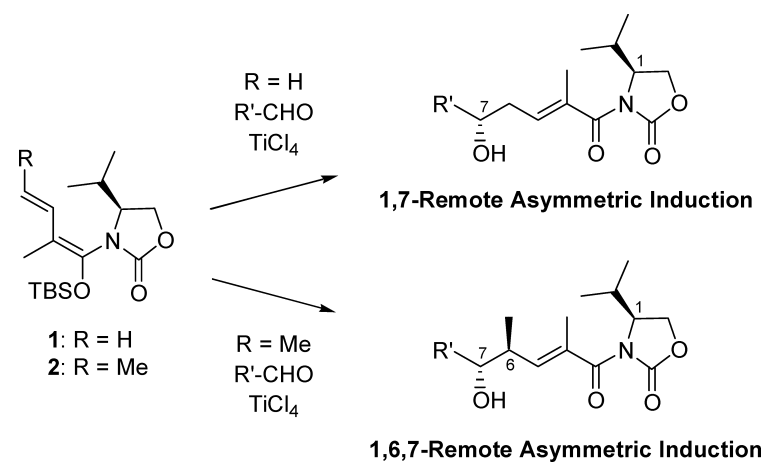

Chart 1. Vinylogous Mukaiyama Aldol Reaction the yields of aldol adducts and shortened the reaction time compared with VMAR of tiglic aldehyde 3d (entry 4). Independently, Hosokawa and Tatsuta et al. reported that the VMAR of ent-2 and tiglic aldehyde $\mathbf{3 d}$ afforded ent-4d in good yield $(82 \%)$ over a prolonged reaction time $(4 \mathrm{~d})$ at low temperature $\left.\left(-60^{\circ} \mathrm{C}\right) .5,11\right)$

Stereochemical determination of the aldol adducts $4 \mathbf{a}$ and 4b were easily achieved by Stille cross-coupling using a catalytic amount of $\mathrm{Pd}(\mathrm{dba})_{2}$ and $\mathrm{Me}_{4} \mathrm{Sn}$ to convert the antiadduct 4d (Chart 2). The spectroscopic data of synthetic $\mathbf{4 d}$

Table 1. VMAR of $\alpha$-Haloenals

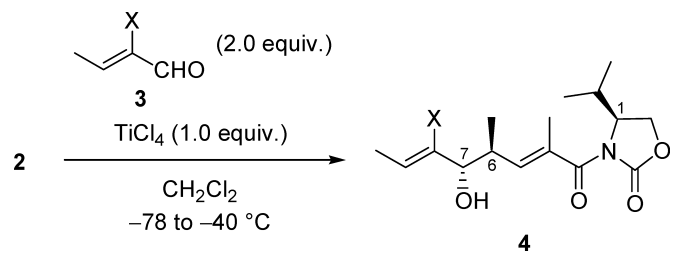

\begin{tabular}{|c|c|c|c|c|c|}
\hline Entry & \multicolumn{2}{|c|}{$\mathrm{X}$} & Time (h) & Yield $(\%)$ & d.r. ${ }^{a)}$ \\
\hline 1 & I & $3 \mathbf{a}$ & 10 & 95 & $>20: 1$ \\
\hline 2 & $\mathrm{Br}$ & $3 b$ & 12 & 88 & $>20: 1$ \\
\hline 3 & $\mathrm{Cl}$ & $3 c$ & 4 & 93 & $>20: 1$ \\
\hline 4 & $\mathrm{Me}$ & $3 d$ & 14 & 76 & $>20: 1$ \\
\hline
\end{tabular}

a) Diastereomeric ratio was determined by ${ }^{1} \mathrm{H}-\mathrm{NMR}$ analysis.
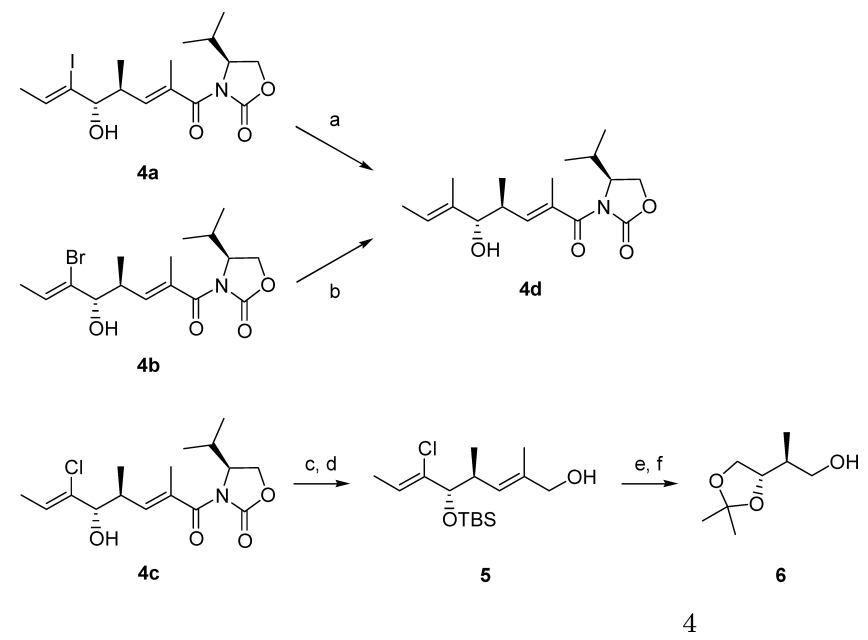

Reagents and conditions: (a) $\mathrm{Me}_{4} \mathrm{Sn}, \mathrm{Pd}(\mathrm{dba})_{2}, \mathrm{Ph}_{3} \mathrm{As}$, HMPA, $60{ }^{\circ} \mathrm{C}, 92 \%$; (b) $\mathrm{Me}_{4} \mathrm{Sn}, \mathrm{Pd}(\mathrm{dba})_{2}, \mathrm{HMPA}, 60^{\circ} \mathrm{C}, 11 \%$ (brsm $72 \%$ ); (c) TBSOTf, $i$-Pr ${ }_{2} \mathrm{NEt}$, $\mathrm{CH}_{2} \mathrm{Cl}_{2}, 0^{\circ} \mathrm{C}, 100 \%$; (d) $\mathrm{NaBH}_{4}, \mathrm{THF}-\mathrm{H}_{2} \mathrm{O}, \mathrm{RT}, 99 \%$; (e) $\mathrm{O}_{3}, \mathrm{CH}_{2} \mathrm{Cl}_{2}-\mathrm{MeOH}$, $-78^{\circ} \mathrm{C}$ then $\mathrm{NaBH}_{4}$, RT, $38 \%$; (f) $p$-TsOH, acetone, RT, quant.

Chart 2. Determination of the Stereochemistry of $\mathbf{4 a}, \mathbf{4 b}$ and $\mathbf{4 c}$ 
Table 2. Pd-Mediated Cross-Coupling with Vinyl Iodide 4a

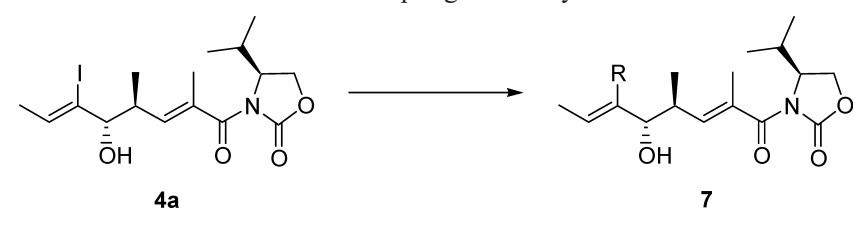

\begin{tabular}{|c|c|c|c|c|}
\hline Entry & Conditions & $\mathrm{R}$ & & $\begin{array}{c}\text { Yield } \\
(\%)\end{array}$ \\
\hline 1 & $\mathrm{Me}_{4} \mathrm{Sn}, \mathrm{Pd}(\mathrm{dba})_{2}, \mathrm{AsPh}_{3}, \mathrm{HMPA}, 60^{\circ} \mathrm{C}$ & $\mathrm{Me}$ & $4 d$ & 92 \\
\hline 2 & $\mathrm{Et}_{2} \mathrm{Zn}, \mathrm{Pd}\left(\mathrm{PPh}_{3}\right)_{4}, \mathrm{THF}, 0{ }^{\circ} \mathrm{C}$ to $\mathrm{RT}$ & Et & $7 \mathbf{a}$ & 72 \\
\hline 3 & $\mathrm{CH}_{2}=\mathrm{CHSnBu}_{3}, \mathrm{Pd}(\mathrm{dba})_{2}, \mathrm{AsPh}_{3}, \mathrm{HMPA}, \mathrm{RT}$ & & $7 \mathbf{b}$ & 96 \\
\hline
\end{tabular}

from $\mathbf{4 a}$ and $\mathbf{4 b}$ agreed with those of the known ent-4d. ${ }^{11)}$ (Synthetic 4d: $[\alpha]_{\mathrm{D}}^{25}-17.3, c=1.09$ in $\mathrm{CH}_{2} \mathrm{Cl}_{2}$, reported ent4d: $[\alpha]_{\mathrm{D}}^{25}+16.5, c=1.16$ in $\mathrm{CH}_{2} \mathrm{Cl}_{2}$ ).

Stereochemistry of the aldol adduct $4 \mathbf{c}$ was determined by transforming to the known compound $\mathbf{6}^{31)}$ After protection of the secondary alcohol with TBSOTf, the chiral auxiliary group was reductively removed with $\mathrm{NaBH}_{4}$ to provide the alcohol 5. Ozonolysis of 5, reduction of the resulting aldehyde, followed by acetal formation gave the known compound $\mathbf{6}$. The spectroscopic data of synthetic $\mathbf{6}$ was identical to those reported for $\mathbf{6}^{31)}$

Next, a series of representative Pd-catalyzed cross-coupling reactions were applied to vinyl iodide $\mathbf{4 a}$ in order to demonstrate the synthetic utility of the present approach (Table 2). Introduction of methyl group to $4 \mathbf{a}$ (entry 1) is already described in Chart 2. Negishi cross-coupling with $\mathrm{Et}_{2} \mathrm{Zn}$ and $\mathrm{Pd}\left(\mathrm{PPh}_{3}\right)_{4}$ afforded ethyl derivative $7 \mathbf{a}$ in $72 \%$ yield (entry 2). Introduction of a vinyl group to $\mathbf{4 a}$ was performed by Stille cross-coupling to afford $\mathbf{7 b}$ in excellent yield (entry 3 ).

In conclusion, we were able to overcome the low reactivity of enals in VMAR by employing $\alpha$-haloenals as substrates. Moreover, the combination of VMAR of $\alpha$-haloenal and well-established Pd-catalyzed cross-coupling has been demonstrated to considerably expand the scope of VMAR.

\section{Experimental}

General Techniques All non-aqueous reactions were carried out under an argon atmosphere in oven-dried of flame-dried glassware. Tetrahydrofuran (THF) and $\mathrm{Et}_{2} \mathrm{O}$ were purchased from Kanto Chemical Co., Inc., Tokyo, Japan in anhydrous grade. $\mathrm{CH}_{2} \mathrm{Cl}_{2}$ was distilled from $\mathrm{CaH}_{2}$ immediately before use. $\mathrm{TiCl}_{4}$ was distilled from granular cupper and stored in ampoules. Diisopropylethylamine was distilled from $\mathrm{CaH}_{2}$ and stored over KOH. Other reagents were used as received. All reactions were monitored by TLC, which was carried out on $0.25 \mathrm{~mm}$ Silica Gel $60 \mathrm{~F}_{254}$ plates (E. Merck). Flash column chromatography separations were performed on PSQ 100B (Fuji Silysia Co., Ltd., Japan). The NMR spectra $\left({ }^{1} \mathrm{H},{ }^{13} \mathrm{C}\right)$ were determined on a Bruker $600 \mathrm{MHz}$ spectrometer (Avance DRX-600) or on a JEOL $400 \mathrm{MHz}$ spectrometer (JNM-LD400), using $\mathrm{CDCl}_{3}$ solutions, unless otherwise noted. Chemical shifts for ${ }^{1} \mathrm{H}-\mathrm{NMR}$ were expressed in parts per million (ppm) downfield from tetramethylsilane $(\delta)$ in duteriochloroform as internal standard and coupling constants $(J)$ are in hertz $(\mathrm{Hz})$. Multiplicities are indicated as: br (broadened), s (singlet), d (doublet), t (triplet), q (quartet), or m (multiplet). Melting points were determined with SHIMADZU MM-2 or Yanaco MP-3S melting point apparatus and were uncorrected. Optical rotations were recorded using $\mathrm{CHCl}_{3}$ or $\mathrm{CH}_{2} \mathrm{Cl}_{2}$ as solvents on a JASCO P-1030 digital polarimeter at room temperature, using sodium D line. Infrared spectra (IR) were recorded on a JASCO FT/IR-410 spectrometer using NaCl (neat) or $\mathrm{KBr}$ pellets (solid), and are reported in wavenumbers $\left(\mathrm{cm}^{-1}\right)$. Mass spectra (MS) were obtained on an Applied Biosystems mass spectrometer (API QSTAR pulsar i) under conditions as High resolution, using poly(ethylene glycol) as internal standard.
General Procedure for Vinylogous Mukaiyama Aldol Reaction of Vinylketene Silyl $\boldsymbol{N}, \boldsymbol{O}$-Acetal $\mathbf{2}$ with $\boldsymbol{\alpha}$-Haloenals To a solution of aldehyde $(1.41 \mathrm{mmol}, 2.0 \mathrm{eq})$ in $\mathrm{CH}_{2} \mathrm{Cl}_{2}(3.5 \mathrm{ml})$, a $1.0 \mathrm{M} \mathrm{TiCl}{ }_{4}$ solution in $\mathrm{CH}_{2} \mathrm{Cl}_{2}(0.70 \mathrm{ml}, 0.705 \mathrm{mmol}, 1.0 \mathrm{eq})$ was added dropwise at $-78^{\circ} \mathrm{C}$. Then a solution of $1(239 \mathrm{mg}, 0.705 \mathrm{mmol})$ in $\mathrm{CH}_{2} \mathrm{Cl}_{2}(3.5 \mathrm{ml})$ was added dropwise into the aldehyde solution at $-78^{\circ} \mathrm{C}$. After stirring for 4 to $16 \mathrm{~h}$ at $-40^{\circ} \mathrm{C}$, the cold reaction mixture was poured into $1: 1$ mixture of saturated $\mathrm{NaHCO}_{3}$ aq. and saturated Rochelle salt aq. $(25 \mathrm{ml})$. The mixture was diluted with $\mathrm{Et}_{2} \mathrm{O}(25 \mathrm{ml})$, and stirred vigorously at room temperature until the white slurry was completely dissolved. The aqueous layer was extracted two times with $\mathrm{Et}_{2} \mathrm{O}$. The combined organic layer was washed with brine, dried over anhydrous $\mathrm{Na}_{2} \mathrm{SO}_{4}$, filtered, and concentrated in vacuo. The residue was purified by column chromatography on silica gel (elution with hexane/AcOEt) to give the aldol adducts.

Aldol adduct 4a: TLC, $R f=0.28$ (hexane/AcOEt $=2: 1$ ). mp $114^{\circ} \mathrm{C} .{ }^{1} \mathrm{H}-$ $\operatorname{NMR}\left(600 \mathrm{MHz}, \mathrm{CDCl}_{3}\right) \delta: 0.86(3 \mathrm{H}, \mathrm{d}, J=6.6 \mathrm{~Hz}), 0.93(3 \mathrm{H}, \mathrm{d}, J=6.9 \mathrm{~Hz})$, $0.94(3 \mathrm{H}, \mathrm{d}, J=7.0 \mathrm{~Hz}), 1.84(3 \mathrm{H}, \mathrm{d}, J=6.4 \mathrm{~Hz}), 2.02(3 \mathrm{H}, \mathrm{d}, J=1.3 \mathrm{~Hz})$, $2.36(1 \mathrm{H}, \mathrm{dqq}, J=4.7,7.0,6.9 \mathrm{~Hz}), 2.94(1 \mathrm{H}, \mathrm{ddq}, J=10.5,9.0,6.6 \mathrm{~Hz}), 3.30$ $(1 \mathrm{H}, \mathrm{dd}, J=9.0,2.4 \mathrm{~Hz}), 3.64(1 \mathrm{H}, \mathrm{d}, J=2.4 \mathrm{~Hz}), 4.20(1 \mathrm{H}, \mathrm{dd}, J=9.1$, $5.6 \mathrm{~Hz}), 4.35(1 \mathrm{H}, \mathrm{dd}, J=9.1,9.1 \mathrm{~Hz}), 4.58(1 \mathrm{H}, \mathrm{ddd}, J=9.1,5.6,4.7 \mathrm{~Hz})$, $5.82(1 \mathrm{H}, \quad \mathrm{dq}, J=10.5, \quad 1.3 \mathrm{~Hz}), \quad 6.02(1 \mathrm{H}, \quad \mathrm{q}, \quad J=6.4, \mathrm{~Hz}) .{ }^{13} \mathrm{C}-\mathrm{NMR}$ $\left(150 \mathrm{MHz}, \mathrm{CDCl}_{3}\right) \delta: 14.3,15.2,16.0,17.8,21.4,28.5,40.0,58.1,63.5$, $81.7,113.9,133.0,133.5,139.3,154.4,171.3 .[\alpha]_{\mathrm{D}}^{24}-26.6(c=1.00$, $\left.\mathrm{CHCl}_{3}\right)$. IR $\left(\mathrm{CHCl}_{3}, \mathrm{~cm}^{-1}\right) 3486,2965,1769,1687,1366,1301,1209$. high resolution (HR)-MS (electrospray ionization (ESI)) $\mathrm{m} / \mathrm{z}$ : 444.0644 (Calcd for $\mathrm{C}_{16} \mathrm{H}_{24} \mathrm{NO}_{4} \mathrm{NaI}[\mathrm{M}+\mathrm{Na}]^{+}$444.0642).

Aldol adduct 4b: TLC, $R f=0.52$ (hexane/AcOEt $=2: 1$ ). ${ }^{1} \mathrm{H}-\mathrm{NMR}$ $\left(600 \mathrm{MHz}, \mathrm{CDCl}_{3}\right) \delta: 0.90(3 \mathrm{H}, \mathrm{d}, J=6.6 \mathrm{~Hz}), 0.93(3 \mathrm{H}, \mathrm{d}, J=6.9 \mathrm{~Hz}), 0.94$ $(3 \mathrm{H}, \mathrm{d}, J=7.0 \mathrm{~Hz}), 1.81(3 \mathrm{H}, \mathrm{d}, J=6.5 \mathrm{~Hz}), 2.01(3 \mathrm{H}, \mathrm{d}, J=1.4 \mathrm{~Hz}), 2.36$ $(1 \mathrm{H}, \mathrm{dqq}, J=4.6,7.0,6.9 \mathrm{~Hz}), 3.02(1 \mathrm{H}, \mathrm{ddq}, J=10.4,8.7,6.6 \mathrm{~Hz}), 3.59$ $(1 \mathrm{H}, \mathrm{d}, J=2.8 \mathrm{~Hz}), 3.79(1 \mathrm{H}, \mathrm{dd}, J=8.7,2.8 \mathrm{~Hz}), 4.20(1 \mathrm{H}, \mathrm{dd}, J=9.0$, $5.5 \mathrm{~Hz}), 4.35(1 \mathrm{H}, \mathrm{dd}, J=9.0,9.0 \mathrm{~Hz}), 4.58(1 \mathrm{H}, \mathrm{ddd}, J=9.0,5.5,4.6 \mathrm{~Hz})$, $5.77(1 \mathrm{H}, \mathrm{dq}, J=10.4,1.4 \mathrm{~Hz}), 6.07(1 \mathrm{H}, \mathrm{q}, J=6.5 \mathrm{~Hz}) .{ }^{13} \mathrm{C}-\mathrm{NMR}(150 \mathrm{MHz}$, $\left.\mathrm{CDCl}_{3}\right) \delta: 14.2,15.2,16.1,16.4,17.8,28.5,38.5,58.1,63.5,80.7,127.6$, 129.6, 132.9, 139.7, 154.4, 171.3. $[\alpha]_{\mathrm{D}}^{24}-16.7\left(c=1.15, \mathrm{CHCl}_{3}\right)$. IR (neat, $\left.\mathrm{cm}^{-1}\right) 3494,2965,2875,1772,1685,1455,1367,1301,1209$. HR-MS (ESI) $m / z: 396.0769$ (Calcd for $\mathrm{C}_{16} \mathrm{H}_{24} \mathrm{NO}_{4} \mathrm{NaBr}[\mathrm{M}+\mathrm{Na}]^{+}$396.0780).

Aldol adduct 4c: TLC, $R f=0.29$ (hexane/AcOEt $=2: 1$ ). ${ }^{1} \mathrm{H}-\mathrm{NMR}$ $\left(600 \mathrm{MHz}, \mathrm{CDCl}_{3}\right) \delta: 0.92(3 \mathrm{H}, \mathrm{d}, J=6.6 \mathrm{~Hz}), 0.93(3 \mathrm{H}, \mathrm{d}, J=7.0 \mathrm{~Hz}), 0.94$ $(3 \mathrm{H}, \mathrm{d}, J=7.1 \mathrm{~Hz}), 1.80(3 \mathrm{H}, \mathrm{d}, J=6.6 \mathrm{~Hz}), 2.00(3 \mathrm{H}, \mathrm{d}, J=1.4 \mathrm{~Hz}), 2.35$ $(1 \mathrm{H}, \mathrm{dqq}, J=4.6,7.1,7.0 \mathrm{~Hz}), 3.02(1 \mathrm{H}$, ddq, $J=10.4,8.8,6.6 \mathrm{~Hz}), 3.54$ $(1 \mathrm{H}, \mathrm{d}, J=3.0 \mathrm{~Hz}), 3.90(1 \mathrm{H}, \mathrm{dd}, J=8.8,3.0 \mathrm{~Hz}), 4.20(1 \mathrm{H}, \mathrm{dd}, J=9.0$, $5.5 \mathrm{~Hz}), 4.35(1 \mathrm{H}, \mathrm{dd}, J=9.0,9.0 \mathrm{~Hz}), 4.58(1 \mathrm{H}, \mathrm{ddd}, J=9.0,5.5,4.6 \mathrm{~Hz})$, $5.75(1 \mathrm{H}, \mathrm{dq}, J=10.4,1.4 \mathrm{~Hz}), 5.85(1 \mathrm{H}, \mathrm{q}, J=6.6 \mathrm{~Hz}) .{ }^{13} \mathrm{C}-\mathrm{NMR}(150 \mathrm{MHz}$, $\left.\mathrm{CDCl}_{3}\right) \delta: 13.7,14.1,15.2,16.1,17.8,28.5,37.7,58.1,63.5,80.0,124.5$, $132.8,134.8,139.9,154.4,171.3 .[\alpha]_{\mathrm{D}}^{24}-14.7\left(c=1.00, \mathrm{CHCl}_{3}\right)$. IR $\left(\mathrm{CHCl}_{3}, \mathrm{~cm}^{-1}\right) 3494,2965,2875,1770,1687,1390,1367,1315,1209$. HRMS (ESI) $m / z$ : 352.1281 (Calcd for $\mathrm{C}_{16} \mathrm{H}_{24} \mathrm{NO}_{4} \mathrm{NaCl}[\mathrm{M}+\mathrm{Na}]^{+} 352.1286$ ).

Aldol adduct 4d: TLC, $R f=0.34$ (hexane/AcOEt $=2: 1$ ). ${ }^{1} \mathrm{H}-\mathrm{NMR}$ $\left(600 \mathrm{MHz}, \mathrm{CDCl}_{3}\right) \delta: 0.82(3 \mathrm{H}, \mathrm{d}, J=6.7 \mathrm{~Hz}), 0.93(3 \mathrm{H}, \mathrm{d}, J=6.8 \mathrm{~Hz}), 0.94$ $(3 \mathrm{H}, \mathrm{d}, J=7.0 \mathrm{~Hz}), 1.64(3 \mathrm{H}, \mathrm{dd}, J=6.6,1.0 \mathrm{~Hz}), 1.67(3 \mathrm{H}, \mathrm{dd}, J=1.0 \mathrm{~Hz})$, $1.98(3 \mathrm{H}, \mathrm{d}, J=1.5 \mathrm{~Hz}), 2.35(1 \mathrm{H}, \mathrm{dqq}, J=4.6,7.0,6.8 \mathrm{~Hz}), 2.75(1 \mathrm{H}, \mathrm{ddq}$, $J=10.3,9.3,6.7 \mathrm{~Hz}), 3.33(1 \mathrm{H}$, br s), $3.67(1 \mathrm{H}, \mathrm{brd}, J=9.3 \mathrm{~Hz}), 4.19(1 \mathrm{H}$, dd, $J=9.0,5.7 \mathrm{~Hz}), 4.35(1 \mathrm{H}, \mathrm{dd}, J=9.0,9.0 \mathrm{~Hz}), 4.58(1 \mathrm{H}, \mathrm{ddd}, J=9.0,5.7$, $4.6 \mathrm{~Hz}), 5.48(1 \mathrm{H}, \mathrm{dq}, J=6.6,1.0 \mathrm{~Hz}), 5.78(1 \mathrm{H}, \mathrm{dq}, J=10.3,1.5 \mathrm{~Hz}) .{ }^{13} \mathrm{C}-$ NMR $\left(150 \mathrm{MHz}, \mathrm{CDCl}_{3}\right) \delta: 10.5,13.1,14.0,15.2,16.1,17.8,28.4,37.8$, $58.1,63.4,82.2,123.7,131.7,134.8,142.0,154.5,171.5 .[\alpha]_{\mathrm{D}}^{24}-17.3$ $\left(c=1.09, \mathrm{CH}_{2} \mathrm{Cl}_{2}\right)$. IR (neat, $\left.\mathrm{cm}^{-1}\right) 3513,2965,2929,2873,1768,1687$, 1367, 1301, 1280, 1211. HR-MS (ESI) m/z: 332.1822 (Calcd for $\left.\mathrm{C}_{17} \mathrm{H}_{27} \mathrm{NO}_{4} \mathrm{Na}[\mathrm{M}+\mathrm{Na}]^{+} 332.1832\right)$.

Alcohol 5: To a solution of $\mathbf{4 c}(38.5 \mathrm{mg}, 0.117 \mathrm{mmol})$ in $\mathrm{CH}_{2} \mathrm{Cl}_{2}(1.2 \mathrm{ml})$, $i$-Pr ${ }_{2} \mathrm{NEt}(0.061 \mathrm{ml}, 0.350 \mathrm{mmol})$ and TBSOTf $(0.064 \mathrm{ml}, 0.280 \mathrm{mmol})$ was added at $0{ }^{\circ} \mathrm{C}$. After stirring for $40 \mathrm{~min}$, the reaction was quenched with $\mathrm{MeOH}$. After stirring further $10 \mathrm{~min}$, the mixture was concentrated in vacuo. The residue was purified by column chromatography on silica gel (elution with hexane/ $\mathrm{AcOEt}=8: 1)$ to give the corresponding TBS ether $(51.8 \mathrm{mg}$, $100 \%$ yield). To a solution of the corresponding TBS ether $(49.7 \mathrm{mg}$, $0.112 \mathrm{mmol})$ in THF $(2.8 \mathrm{ml}), \mathrm{NaBH}_{4}(29.6 \mathrm{mg}, 0.783 \mathrm{mmol})$ in water $(0.6 \mathrm{ml})$ was added at $0{ }^{\circ} \mathrm{C}$. After stirring for $3 \mathrm{~h}$ at r.t., the reaction was diluted with water and $\mathrm{Et}_{2} \mathrm{O}$. The aqueous layer was extracted two times with $\mathrm{Et}_{2} \mathrm{O}$. The combined organic layer was washed with brine, dried over anhydrous $\mathrm{Na}_{2} \mathrm{SO}_{4}$, filtered, and concentrated in vacuo. The residue was purified by column chromatography on silica gel (elution with hexane/AcOEt $=8: 1$ ) to give alcohol 5 (35.2 mg, 99\% yield). Alcohol 5: colorless oil. TLC, 
$R f=0.59$ (hexane/AcOEt $=2: 1) .{ }^{1} \mathrm{H}-\mathrm{NMR}\left(600 \mathrm{MHz}, \mathrm{CDCl}_{3}\right) \delta:-0.01(3 \mathrm{H}$, s), $0.01(3 \mathrm{H}, \mathrm{s}), 0.86(9 \mathrm{H}, \mathrm{s}), 0.88(3 \mathrm{H}, \mathrm{d}, J=6.9 \mathrm{~Hz}), 1.16(1 \mathrm{H}, \mathrm{t}, J=6.2 \mathrm{~Hz})$, $1.67(3 \mathrm{H}, \mathrm{d}, J=1.3 \mathrm{~Hz}), 1.73(3 \mathrm{H}, \mathrm{d}, J=6.6 \mathrm{~Hz}), 2.80(1 \mathrm{H}, \mathrm{ddq}, J=9.7,6.5$, $6.9 \mathrm{~Hz}), 3.88(1 \mathrm{H}, \mathrm{d}, J=6.5 \mathrm{~Hz}), 3.99(2 \mathrm{H}, \mathrm{d}, J=6.2 \mathrm{~Hz}), 5.23(1 \mathrm{H}, \mathrm{dq}$, $J=9.7,1.3 \mathrm{~Hz}), 5.71(1 \mathrm{H}, \mathrm{q}, J=6.6 \mathrm{~Hz}) \cdot{ }^{13} \mathrm{C}-\mathrm{NMR}\left(150 \mathrm{MHz}, \mathrm{CDCl}_{3}\right) \delta$ $-5.1,-4.8,13.4,14.1,17.4,18.1,25.7,36.4,69.1,80.5,121.5,128.3$, 135.4, 136.8. $[\alpha]_{\mathrm{D}}^{21}-15.2\left(c=1.02, \mathrm{CHCl}_{3}\right)$; IR (neat, $\left.\mathrm{cm}^{-1}\right) 3328,2958$, 2929, 2857, 1660, 1461, 1255, 1095, 1006. HR-MS (ESI) $m / z: 341.1658$ (Calcd for $\mathrm{C}_{16} \mathrm{H}_{31} \mathrm{O}_{2} \mathrm{NaSiCl}[\mathrm{M}+\mathrm{Na}]^{+} 341.1674$ ).

Alcohol 6: Alcohol $5(12.1 \mathrm{mg}, 0.0379 \mathrm{mmol})$ was diluted in $1: 1$ $\mathrm{CH}_{2} \mathrm{Cl}_{2} / \mathrm{MeOH}(2.0 \mathrm{ml})$ and cooled to $-78^{\circ} \mathrm{C}$. Ozone was bubbled through the solution until a blue tint was observed (about $3 \mathrm{~min}$ ), and then argon was passed through the solution for $15 \mathrm{~min}$, after which $\mathrm{NaBH}_{4}(11.5 \mathrm{mg}$, $0.303 \mathrm{mmol}$ ) was added. After stirring for $8 \mathrm{~h}$ at r.t., the reaction was quenched with saturated $\mathrm{NH}_{4} \mathrm{Cl}$ aq. The aqueous layer was extracted two times with $\mathrm{Et}_{2} \mathrm{O}$. The combined organic layer was washed with brine, dried over anhydrous $\mathrm{Na}_{2} \mathrm{SO}_{4}$, filtered, and concentrated in vacuo. The residue was purified by column chromatography on silica gel (elution with hexane/AcOEt $=2: 1)$ to give the corresponding diol $(3.4 \mathrm{mg}, 38 \%$ yield). To a solution of the corresponding diol $(2.2 \mathrm{mg}, 9.39 \mathrm{mmol})$ in acetone $(0.5 \mathrm{ml})$, $p$ - $\mathrm{TsOH}(0.9 \mathrm{mg}, 4.69 \mathrm{mmol})$ was added at r.t. After stirring for $16 \mathrm{~h}$, the reaction was neutralized with saturated $\mathrm{NaHCO}_{3}$ aq. The aqueous layer was extracted two times with $\mathrm{Et}_{2} \mathrm{O}$. The combined organic layer was dried over anhydrous $\mathrm{MgSO}_{4}$, filtered, and concentrated in vacuo. The residue was purified by column chromatography on silica gel (elution with petroleum ether/ether $=4: 1)$ to give alcohol $6(1.5 \mathrm{mg}$, quantitative yield). Alcohol 6 : colorless oil. TLC, $R f=0.39$ (hexane/AcOEt $=1: 1)$. ${ }^{1} \mathrm{H}-\mathrm{NMR}(600 \mathrm{MHz}$, $\left.\mathrm{CDCl}_{3}\right) \delta: 0.83(3 \mathrm{H}, \mathrm{d}, J=7.0 \mathrm{~Hz}), 1.37(3 \mathrm{H}, \mathrm{s}), 1.42(3 \mathrm{H}, \mathrm{s}), 1.89-1.82$ $(1 \mathrm{H}, \mathrm{m}), 2.75(1 \mathrm{H}, \mathrm{dd}, J=8.6,2.8 \mathrm{~Hz}), 3.66(1 \mathrm{H}, \mathrm{dd}, J=8.1,7.5 \mathrm{~Hz}), 3.69-$ $3.59(2 \mathrm{H}, \mathrm{m}), 3.95(1 \mathrm{H}$, ddd, $J=8.8,7.5,6.1 \mathrm{~Hz}), 4.10(1 \mathrm{H}, \mathrm{dd}, J=8.1$, $6.1 \mathrm{~Hz}) .{ }^{13} \mathrm{C}-\mathrm{NMR}\left(150 \mathrm{MHz}, \mathrm{CDCl}_{3}\right) \delta: 13.1,25.7,26.7,39.2,67.5,68.8$, 80.9, 109.4. $[\alpha]_{\mathrm{D}}^{22}+30\left(c=0.027, \mathrm{CHCl}_{3}\right)$. IR $\left(\mathrm{CHCl}_{3}, \mathrm{~cm}^{-1}\right) 2921,2852$, 2042, 1070, 1045, 804. HR-MS (ESI) $m / z$ : 183.0993 (Calcd for $\mathrm{C}_{8} \mathrm{H}_{16} \mathrm{O}_{3} \mathrm{Na}$ $[\mathrm{M}+\mathrm{Na}]^{+}$183.0997).

Methyl derivative $4 \mathbf{d}$ : To a solution of iodide $4 \mathbf{a}(30.0 \mathrm{mg}, 0.071 \mathrm{mmol})$ in degassed HMPA $(0.8 \mathrm{ml}), \mathrm{Me}_{4} \mathrm{Sn}(0.04 \mathrm{ml}, 0.285 \mathrm{mmol}), \mathrm{Pd}(\mathrm{dba})_{2}(4.1 \mathrm{mg}$, $0.007 \mathrm{mmol})$ and $\mathrm{AsPh}_{3}(8.7 \mathrm{mg}, 0.028 \mathrm{mmol})$ were added respectively at r.t. After stirring for $40 \mathrm{~min}$ at $60^{\circ} \mathrm{C}$, the reaction mixture was poured into $\mathrm{H}_{2} \mathrm{O}$. The mixture was diluted with $\mathrm{Et}_{2} \mathrm{O}$, and the aqueous layer was extracted two times with $\mathrm{Et}_{2} \mathrm{O}$. The combined organic layer was washed with brine, dried over anhydrous $\mathrm{Na}_{2} \mathrm{SO}_{4}$, filtered, and concentrated in vacuo. The residue was purified by column chromatography on silica gel (elution with hexane/ $\mathrm{AcOEt}=6: 1)$ to give $\mathbf{4 d}(20.2 \mathrm{mg}, 92 \%$ yield $)$.

Ethyl derivative $7 \mathbf{a}$ : To a solution of iodide $4 \mathbf{a}(10.7 \mathrm{mg}, 0.025 \mathrm{mmol})$ in degassed HMPA $(0.5 \mathrm{ml}), \mathrm{Pd}(\mathrm{dba})_{2}(1.5 \mathrm{mg}, 0.0025 \mathrm{mmol})$ and $\mathrm{AsPh}_{3}$ $(3.1 \mathrm{mg}, 0.010 \mathrm{mmol})$ were added respectively at r.t. Then a $1.08 \mathrm{M} \mathrm{Et}_{2} \mathrm{Zn} \mathrm{so}-$ lution in hexane $(0.094 \mathrm{ml}, 0.102 \mathrm{mmol})$ was added dropwise at $0{ }^{\circ} \mathrm{C}$. After stirring for $2 \mathrm{~h}$ at r.t., the reaction mixture was quenched with saturated $\mathrm{NH}_{4} \mathrm{Cl}$ aq. The aqueous layer was extracted two times with $\mathrm{Et}_{2} \mathrm{O}$. The combined organic layer was washed with brine, dried over anhydrous $\mathrm{Na}_{2} \mathrm{SO}_{4}$, filtered, and concentrated in vacuo. The residue was purified by column chromatography on silica gel (elution with hexane/AcOEt $=6: 1$ ) to give 7a (5.9 mg, $72 \%$ yield). Ethyl derivative 7a: TLC, $R f=0.38$ (hexane/AcOEt $=$ 2:1). ${ }^{1} \mathrm{H}-\mathrm{NMR}\left(600 \mathrm{MHz}, \mathrm{CDCl}_{3}\right) \delta: 0.86(3 \mathrm{H}, \mathrm{d}, J=6.6 \mathrm{~Hz}), 0.93(3 \mathrm{H}, \mathrm{d}$, $J=6.9 \mathrm{~Hz}), 0.94(3 \mathrm{H}, \mathrm{d}, J=7.0 \mathrm{~Hz}), 1.08(3 \mathrm{H}, \mathrm{t}, J=7.6 \mathrm{~Hz}), 1.67(3 \mathrm{H}, \mathrm{d}$ $J=6.8 \mathrm{~Hz}), 1.98(3 \mathrm{H}, \mathrm{d}, J=1.4 \mathrm{~Hz}), 2.22-2.11(2 \mathrm{H}, \mathrm{m}), 2.35$ (1H, dqq., $J=4.6,7.0,6.9 \mathrm{~Hz}), 2.76(1 \mathrm{H}, \mathrm{ddq}, J=10.3,9.2,6.6 \mathrm{~Hz}), 3.30(1 \mathrm{H}, \mathrm{d}$, $J=1.9 \mathrm{~Hz}), 3.70(1 \mathrm{H}$, br d, $J=9.2 \mathrm{~Hz}), 4.19(1 \mathrm{H}, \mathrm{dd}, J=9.0,5.8 \mathrm{~Hz}), 4.34$ $(1 \mathrm{H}, \mathrm{dd}, J=9.0,9.0 \mathrm{~Hz}), 4.58(1 \mathrm{H}, \mathrm{ddd}, J=9.0,5.8,4.6 \mathrm{~Hz}), 5.47(1 \mathrm{H}, \mathrm{q}$, $J=6.8, \mathrm{~Hz}), 5.80(1 \mathrm{H}, \mathrm{dq}, J=10.3,1.4 \mathrm{~Hz}) \cdot{ }^{13} \mathrm{C}-\mathrm{NMR}\left(150 \mathrm{MHz}, \mathrm{CDCl}_{3}\right) \delta$ : $13.0,13.98,14.01,15.2,16.5,17.8,19.8,28.5,38.5,58.1,63.5,81.8,123.5$, $131.7,141.0,142.2,154.4,171.5 .[\alpha]_{\mathrm{D}}^{24}-62\left(c=0.013, \mathrm{CHCl}_{3}\right)$. IR (neat, $\mathrm{cm}^{-1}$ ) 3515, 2964, 2931, 2873, 1772, 1685, 1390, 1365, 1301, 1280, 1209. HR-MS (ESI) $m / z$ : 346.1996 (Calcd for $\mathrm{C}_{18} \mathrm{H}_{29} \mathrm{NO}_{4} \mathrm{Na}[\mathrm{M}+\mathrm{Na}]^{+} 346.1988$ ).

Vinyl derivative $7 \mathbf{b}$ : To a solution of iodide $4 \mathbf{a}(30.2 \mathrm{mg}, 0.072 \mathrm{mmol})$ in degassed HMPA $(0.8 \mathrm{ml})$, vinyl(tributyl)tin $(45.5 \mathrm{mg}, 0.143 \mathrm{mmol}), \mathrm{Pd}(\mathrm{dba})_{2}$ $(4.1 \mathrm{mg}, 0.007 \mathrm{mmol})$ and $\mathrm{AsPh}_{3}(8.8 \mathrm{mg}, 0.029 \mathrm{mmol})$ were added respectively at r.t. After stirring for $10 \mathrm{~min}$ at r.t., the reaction mixture was poured into $\mathrm{H}_{2} \mathrm{O}$. The mixture was diluted with $\mathrm{Et}_{2} \mathrm{O}$, and the aqueous layer was extracted two times with $\mathrm{Et}_{2} \mathrm{O}$. The combined organic layer was washed with brine, dried over anhydrous $\mathrm{Na}_{2} \mathrm{SO}_{4}$, filtered, and concentrated in vacuo. The residue was purified by column chromatography on silica gel (elution with hexane, then hexane/AcOEt $=8: 1)$ to give $7 \mathbf{b}(22.2 \mathrm{mg}, 96 \%$ yield $)$. Vinyl derivative 7b: TLC, $R f=0.48$ (hexane/AcOEt $=2: 1) .{ }^{1} \mathrm{H}-\mathrm{NMR}(600 \mathrm{MHz}$,
$\left.\mathrm{CDCl}_{3}\right) \delta: 0.87(3 \mathrm{H}, \mathrm{d}, J=6.7 \mathrm{~Hz}), 0.93(3 \mathrm{H}, \mathrm{d}, J=6.9 \mathrm{~Hz}), 0.94(3 \mathrm{H}, \mathrm{d}$, $J=7.0 \mathrm{~Hz}), 1.79(3 \mathrm{H}, \mathrm{d}, J=7.0 \mathrm{~Hz}), 1.96(3 \mathrm{H}, \mathrm{d}, J=1.5 \mathrm{~Hz}), 2.35(1 \mathrm{H}$, dqq, $J=4.6,7.0,6.9 \mathrm{~Hz}), 2.94(1 \mathrm{H}, \mathrm{ddq}, J=10.4,8.7,6.7 \mathrm{~Hz}), 3.37(1 \mathrm{H}, \mathrm{d}$, $J=2.4 \mathrm{~Hz}), 3.97(1 \mathrm{H}, \mathrm{dd}, J=8.7,2.4 \mathrm{~Hz}), 4.20(1 \mathrm{H}, \mathrm{dd}, J=9.0,5.7 \mathrm{~Hz}), 4.35$ $(1 \mathrm{H}, \mathrm{dd}, J=9.0,9.0 \mathrm{~Hz}), 4.59(1 \mathrm{H}, \mathrm{ddd}, J=9.0,5.7,4.6 \mathrm{~Hz}), 5.24(1 \mathrm{H}, \mathrm{br}$, $J=11.5 \mathrm{~Hz}), 5.51(1 \mathrm{H}, \mathrm{dd}, J=17.8,1.7 \mathrm{~Hz}), 5.73(1 \mathrm{H}, \mathrm{q}, J=7.0 \mathrm{~Hz}), 5.83$ $(1 \mathrm{H}, \mathrm{dq}, J=10.4,1.3 \mathrm{~Hz}), 6.58(1 \mathrm{H}, \mathrm{dd}, J=17.8,11.5 \mathrm{~Hz}) .{ }^{13} \mathrm{C}-\mathrm{NMR}$ $\left(150 \mathrm{MHz}, \mathrm{CDCl}_{3}\right) \delta: 13.5,14.0,15.2,16.5,17.8,28.5,39.1,58.1,63.5$, $79.0,115.9,126.9,131.2,131.7,137.5,142.1,154.5,171.5 .[\alpha]_{\mathrm{D}}^{24}-51$ $\left(c=0.19, \mathrm{CHCl}_{3}\right)$. IR (neat, $\left.\mathrm{cm}^{-1}\right) 3505,2965,2929,2875,1770,1685$, 1390, 1367, 1301, 1211. HR-MS (ESI) $m / z$ : 344.1844 (Calcd for $\mathrm{C}_{18} \mathrm{H}_{27} \mathrm{NO}_{4} \mathrm{Na}[\mathrm{M}+\mathrm{Na}]^{+}$344.1832).

Acknowledgement This research was supported in part by a Grant-inAid for Scientific Research (B) (KAKENHI No. 18390010) from the Japan Society for the Promotion of Science.

\section{References and Notes}

1) Shirokawa S.-i., Kamiyama M., Nakamura T., Okada M., Nakazaki A., Hosokawa S., Kobayashi S., J. Am. Chem. Soc., 126, 13604-13605 (2004).

2) Shinoyama M., Shirokawa S.-i., Nakazaki A., Kobayashi S., Org. Lett., 11, 1277-1280 (2009).

3) Yamaoka M., Nakazaki A., Kobayashi S., Tetrahedron Lett., 51, 287 289 (2010).

4) Hosokawa S., Ogura T., Togashi H., Tatsuta K., Tetrahedron Lett., 46 , 333 -337 (2005).

5) Hosokawa S., Yokota K., Imamura K., Suzuki Y., Kawarasaki M., Tatsuta K., Tetrahedron Lett., 47, 5415-5418 (2006).

6) Hosokawa S., Kuroda S., Imamura K., Tasuta K., Tetrahedron Lett., 47, 6183-6186 (2006).

7) Jiang X., Liu B., Lebreton S., De Brabander J. K., J. Am. Chem. Soc., 129, 6386-6387 (2007).

8) Nicolaou K. C., Guduru R., Sun Y.-P., Banerji B., Chen D. Y.-K., Angew. Chem. Int. Ed., 46, 5896-5900 (2007).

9) Nicolaou K. C., Sun Y.-P., Guduru R., Banerji B., Chen D. Y.-K., J. Am. Chem. Soc., 130, 3633-3644 (2008).

10) Schmauder A., Müller S., Maier M. E., Tetrahedron, 64, 6263-6269 (2008).

11) Hosokawa S., Yokota K., Imamura K., Suzuki Y., Kawarasaki M., Tatsuta K., Asian J. Chem., 3, 1415-1421 (2008).

12) Lipshutz B. H., Amorelli B., J. Am. Chem. Soc., 131, 1396-1397 (2009).

13) Wang L., Gong J., Deng L., Xiang Z., Chen Z., Wang Y., Chen J., Yang Z., Org. Lett., 11, 1809-1812 (2009).

14) Hosokawa S., Mukaeda Y., Kawahara R., Tatsuta K., Tetrahedron Lett., 50, 6701-6704 (2009).

15) Hosokawa S., Matsushita K., Tokimatsu S., Toriumi T., Suzuki Y., Tatsuta K., Tetrahedron Lett., 51, 5532-5536 (2010).

16) Paterson I., Kan S. B. J., Gibson L. J., Org. Lett., 12, 3724-3727 (2010).

17) Wang L., Xi Y., Yang S., Zhu R., Liang Y., Chen J., Yang Z., Org. Lett., 13, 74-77 (2011).

18) Tatsuta K., Hosokawa S., Chem. Rev., 105, 4707-4729 (2005).

19) Tatsuta K., Hosokawa S., Chem. Rec., 6, 217-233 (2006).

20) Hosokawa S., Tatsuta K., Mini-Rev. Org. Chem., 5, 1-18 (2008).

21) Nakamura T., Shirokawa S.-i., Hosokawa S., Nakazaki A., Kobayashi S., Org. Lett., 8, 677-679 (2006).

22) Shirokawa S.-i., Shinoyama M., Ooi I., Hosokawa S., Nakazaki A., Kobayashi S., Org. Lett., 9, 849-852 (2007).

23) Yamaoka M., Fukatsu Y., Nakazaki A., Kobayashi S., Tetrahedron Lett., 50, 3849-3852 (2009).

24) Yamaoka M., Nakazaki A., Kobayashi S., Tetrahedron Lett., 50, $6764-6768$ (2009).

25) Matsui R., Seto K., Sato Y., Suzuki T., Nakazaki A., Kobayashi S., Angew. Chem. Int. Ed., 50, 680-683 (2011).

26) Krafft M. E., Cran J. W., Synlett, 8, 1263-1266 (2005).

27) Lutjens H., Nowotny S., Knochel P., Tetrahedron Asymmetry, 6, 2675-2678 (1995).

28) Kamigata N., Satoh T., Yoshida M., Bull. Chem. Soc. Jpn., 61, 449454 (1988).

29) Ley S. V., Whittle A. J., Tetrahedron Lett., 22, 3301-3304 (1981).

30) Especially, the electron-withdrawing ability of chloro group probably shortened the reaction time in the VMAR of $\alpha$-chloroenal $3 \mathbf{c}$.

31) Nicolaou K. C., Papahatjis D. P., Claremon D. A., Magolda R. L., Dolle R. E., J. Org. Chem., 50, 1440-1456 (1985). 